INPLASY

PROTOCOL

To cite: Moretto et al.

Complications and adverse

effects related to the

hyaluronic acid facial filling

with aesthetic purposes: a

systematic review. Inplasy

protocol 202150069. doi:

10.37766/inplasy2021.5.0069

Received: 17 May 2021

Published: 17 May 2021

Corresponding author:

Gabriela Moretto

gabriela@odontobiz.com.br

Author Affiliation:

ILAPEO

Support: None.

Review Stage at time of this submission: Data extraction.

Conflicts of interest:

None declared.

\section{Complications and adverse effects related to the hyaluronic acid facial filling with aesthetic purposes: a systematic review}

Moretto, G1; Cardoso, JM2; Shimizu, IA3; Silva, RD4; Shimizu, $\mathrm{RH}^{5}$; Granjeiro, JM ${ }^{6}$.

Review question / Objective: Which complications and adverse effects are related to the use of hyaluronic acid fillers for facial aesthetic purposes in adults?

Condition being studied: Bring awareness of the possible side effects and complications to professionals using hyaluronic acid fillers on the patient's face for aesthetic purpose.

Information sources: Data bases: PubMed/MEDLINE, LILACS; Web of Science, Scopus, EBSCO; Grey literature Report; Additional Search: Hand-searches of reference list from included studies and experts.

INPLASY registration number: This protocol was registered with the International Platform of Registered Systematic Review and Meta-Analysis Protocols (INPLASY) on 17 May 2021 and was last updated on 17 May 2021 (registration number INPLASY202150069).

\section{INTRODUCTION}

Review question / Objective: Which complications and adverse effects are related to the use of hyaluronic acid fillers for facial aesthetic purposes in adults?

Condition being studied: Bring awareness of the possible side effects and complications to professionals using hyaluronic acid fillers on the patient's face for aesthetic purpose

\section{METHODS}

Search strategy: ("esthetics procedures" OR "Facial Esthetic procedures" OR "injections" OR "rejuvenation" OR 
"aesthetics" OR "aesthetic medicine" OR "Facial Esthetic") AND ("Hyaluronic Acid" OR "Hialuronan" OR "dermal fillers" OR "skin filler" OR "skin fillers") AND ("adverse effects").

Participant or population: Adults.

Intervention: Facial hyaluronic acid fillers.

Comparator: None.

Study designs to be included: RCTs, Clinical trial.

Eligibility criteria: Studies that present one or more complications or adverse effects caused by the facial hyaluronic acid fillers for aesthetic purposes.

Information sources: Data bases: PubMed/ MEDLINE, LILACS; Web of Science, Scopus, EBSCO; Grey literature Report; Additional Search: Hand-searches of reference list from included studies and experts.

Main outcome(s): Complications and adverse effects related to the hyaluronic acid facial filling with aesthetic purposes.

Data management: Rayyan.

Quality assessment / Risk of bias analysis: RCT studies - The Cochrane Collaboration's tool for assessing risk of bias. Nonrandomized studies - Robins-I or Minors. The level of evidence will be analyzed by using GRADE.

Strategy of data synthesis: Where appropriate, for continuous data mean difference, and for dichotomous data relative risk (RR) values(with 95\% confidence interval) will be calculated. In addition, the contribution weight of studies and their statistical heterogeneity will be also calculated. For statistical significance, a value of $p<0.05$ will be used.

Subgroup analysis: None.

Sensitivity analysis: Two independent reviewers (1R and $2 R$ ) will select the included articles. First, in phase-one, both reviewers will read titles and abstracts independently while applying the eligibility criteria. Second, in phase-two, the same two reviewers (1R and $2 R$ ) will perform a full-text reading while applying the election criteria. In both phases, all the retrieved information will be crosschecked by the third review (3R). Final selection will be always based on the full-text of the publication.

Language: English.

Country(ies) involved: Brazil.

Keywords: Hyaluronic Acid, dermal fillers, skin fillers, adverse effects, rejuvenation.

Contributions of each author:

Author 1 - Gabriela Moretto.

Email: gabriela@odontobiz.com.br

Author 2 - Janderson Cardoso.

Email: jjcardoso@uol.com.br

Author 3 - Isabela Shimizu.

Email: isashimizu@hotmail.com

Author 4 - Ricarda Silva.

Author 5 - Roberto Shimizu.

Author 6 - José Mauro Granjeiro. 